\title{
EL INTERACCIONISMO SIMBÓLICO EN ITALIA Y ESPAÑA: UN CAMINO POR RECORRER
}

\author{
THE SYMBOLIC INTERACTIONISM IN ITALY AND SPAIN: ONE ROAD \\ TO GO
}

\author{
Giuliano Tardivo \\ Maximiliano Fernández Fernández \\ Universidad Rey Juan Carlos, Madrid. España/Spain \\ giuliano.tardivo@urjc.es \\ maximiliano.fernandez@urjc.es
}

Recibido/Received: 27/03/2014

Modificado/Modified: 30/05/2014

Aceptado/Accepted: 27/06/2014

\section{RESUMEN}

En el presente texto, después de haber intentado delimitar el concepto de interaccionismo simbólico y haber recorrido rápidamente el origen de esta escuela y las aportaciones de los principales sociólogos de esta corriente, nacida en EE.UU., intentamos averiguar cuál es su presencia, en Italia y en España, en la sociología actual (en revistas, manuales e investigaciones) y si en poetas y autores literarios, como Gozzano y Pirandello, podemos detectar puntos de encuentro con la sociología interaccionista. Para llevar a cabo esta investigación hemos utilizado una metodología comparativo-descriptiva, con técnicas de recogida de los datos, el análisis de documentos (de tipo cuantitativo y sobre todo análisis de contenido de tipo cualitativo). Los resultados demuestran que es una escuela sociológica que ha tenido hasta el momento poco espacio en Italia y en España en comparación con otros países.

\section{PALABRAS CLAVE}

Interaccionismo simbólico, Mead, Goffman, Italia, España, Pirandello, Gozzano

\section{SUMARIO}

1. Introducción. Objetivos y métodos. 2. Un intento de delimitación conceptual: ¿es posible dar una definición de interaccionismo simbólico? 3. ¿Existe un corpus común entre los autores del interaccionismo? El Manifiesto de Blumer como punto de partida. 4. Interaccionismo simbólico y literatura: ¿Pirandello y Gozzano como Goffman? 5. El interaccionismo en las revistas italianas y españolas: ¿Presencia o ausencia? 6. Los manuales sobre el interaccionismo simbólico y las investigaciones con el método de Blumer y Goffman en Italia y en España. 7. Resultados y conclusiones. Bibliografía.

\footnotetext{
ABSTRACT

In the present text, after describing what is known as symbolic interactionism and looking over this school of thought origins swiftly, as well as the main sociologists contributions to this current, born in the USA, we will try to figure out how it has influenced Spanish and Italian sociologist culture (media, manuals and researchers) and even if in poets and literary authors, as Gozzano and Pirandello, we can detect a meeting point with the interacionist sociology. To carry out this research, we have used a comparative-descriptive methodology, with techniques of data collection, document analysis
} 
(quantitative and especially qualitative content analysis types). The results show that it is a sociological school that has had compared to other countries, little spaces in Italy and Spain until this moment.

\section{KEYWORDS}

Symbolic interactionism, Mead, Goffman, Italy, Spain, Pirandello, Gozzano.

\section{CONTENTS}

1. Introduction. Objetives and methods. 2. An attempt of conceptual definition. Is it possible to define symbolic interactionism? 3. Is there a common corpus between the interactionist authors? The Blumer Manifesto as a starting point. 4. Symbolic interactionism and literature: Pirandello and Gozano as Goffman? 5. The interactionism in the Italian and Spanish journals: Presence or absence? 6. Manuals on symbolic interactionism and research with the Blumer and Goffman method in Italy and in Spain. 7. Results and conclusions. References.

\section{INTRODUCCIÓN: OBJETIVOS Y MÉTODOS}

El interaccionismo simbólico es una escuela que surgió en EE.UU. y no en el ámbito europeo, como ocurrió con otras corrientes sociológicas. Su influencia en el mundo anglosajón es conocida, pero menos en Europa del Sur.

El objetivo del presente texto es contestar a la siguiente pregunta de investigación: “iqué presencia y qué influencia ha tenido y tiene el interaccionismo simbólico, respecto a otras escuelas sociológicas, en la sociología y, más en general, en las culturas, italiana y española?" La principal hipótesis o suposición que hemos formulado al principio y que intentamos confirmar o refutar a partir de los documentos y de la bibliografía tomada en consideración es la siguiente: el interaccionismo simbólico ha tenido por lo general una influencia limitada en Italia y en España, en comparación con otras corrientes de pensamiento sociológico.

Además de esta cuestión fundamental, nos hemos hecho otras preguntas secundarias, relacionadas con el mismo problema de investigación. Por ejemplo, si es posible establecer una relación, unas nuevas afinidades electivas, entre autores del interaccionismo simbólico y escritores y poetas, como Gozzano y Pirandello, como se ha hecho en el pasado con Weber y Kafka (González García, 1989) o con Marx y Dickens. En definitiva, si algunas temáticas propias del interaccionismo simbólico, como la atención por la interacción en la vida cotidiana, la marginalidad, la desviación y el interés hacia la identidad cambiante de los sujetos, han encontrado en la cultura italiana y española espacio en la poesía y en los textos literarios, más allá de la sociología propiamente dicha.

Para contestar a estas preguntas y contrastar estas hipótesis, nos hemos centrado en:

1. Intentar definir qué se entiende por "interaccionismo simbólico", describiendo así el marco teórico de referencia, a fin de delimitar este concepto básico.

2. Analizar, con los límites espaciotemporales propios de un artículo y de una investigación breve, la literatura crítica, más que las fuentes directas, sobre Gozzano y Pirandello.

3. Analizar las principales revistas españolas e italianas de sociología para averiguar si el número y la calidad de los artículos relacionados con el interaccionismo simbólico puede ser considerado significativo o poco relevante. Recordamos estudios metodológicamente parecidos llevados a cabo por otros sociólogos, como Oromaner, que realizó una investigación para averiguar cuáles eran los sociólogos que más aparecían en las revistas de 
1958 a 1968 y así comprobar el rumbo de la sociología inglesa y norteamericana en esa época (Picó López, 1997:104). Nosotros intentamos utilizar a continuación la misma metodología investigadora para comprobar la presencia y la influencia del interaccionismo simbólico en Italia y en España.

4. Hemos tomado en consideración también los manuales publicados en Italia y en España sobre esta escuela sociológica y algunas investigaciones publicadas recientemente y llevadas a cabo con la misma metodología utilizada por los interaccionistas, sobre todo a través de la técnica de la observación participante.

Por consiguiente, hemos utilizado, cuando ha sido necesario, el método comparativodescriptivo -comparar dos objetos, Italia y España, en relación a una propiedad o característica: el interaccionismo simbólico- a fin de delimitar y desmenuzar los conceptos, contestar a las preguntas y contrastar las hipótesis, el análisis de documentos, es decir, la literatura crítica y las fuentes primarias y secundarias. Nos hemos centrado, dado el objetivo que hemos fijado al principio de la investigación, en analizar sobre todo las fuentes indirectas -libros y artículos de revistas- publicadas en lengua italiana y española. Esta breve investigación se justifica por el hecho de que hemos detectado que no abundan los estudios teóricos sobre esta escuela, a pesar de su relevante fertilidad teórica y hemos intentado por consiguiente averiguar si esta suposición tiene fundamentos o no.

\section{UN INTENTO DE DELIMITACIÓN CONCEPTUAL: ¿ES POSIBLE DAR UNA DEFINICIÓN DE INTERACCIONISMO SIMBÓLICO?}

Intentamos en este apartado definir qué es el interaccionismo simbólico, presentar esta escuela sociológica, el debate teórico sobre los orígenes y los problemas conceptuales relacionados.

Si leyéramos solamente un libro de metodología, como el de Corbetta (1999:32), podríamos pensar que los padres del interaccionismo simbólico, así como de toda la perspectiva microsociológica, fuesen en realidad Dilthey y Weber, lo cual sería una versión sesgada del origen de esta escuela, dado que en Weber hay cierta atención también hacia los fenómenos colectivos y macrosociales (Álvaro Estramiana et al., 2007b:42). Weber, cuya importancia en la sociología no puede ser cuestionada, ha sido considerado de hecho prácticamente padre de todo: de la sociología, de la sociología del conflicto (Collins, 1996:61), de la sociología comprensiva, y de toda la sociología contemporánea, que es en gran parte o weberiana o durkheimiana, o integradora de ambas perspectivas (interaccionismo simbólico estructural y neofuncionalismo); así como en ciertos momentos se le ha visto como exponente de relieve del liberalismo de Weimar o incluso profeta del nazismo (Zabludovsky Kuper, 2009:48) y de la burocratización del mundo. La sociología weberiana tiene un cierto nivel psicológico, desconocido en otros sociólogos. Weber nunca reduce los hechos sociales -siempre complejos y pluricausales- a probabilidades estadísticas (De Nardis, 2011:98). Esta es una de sus posibles relaciones directas con los sociólogos del interaccionismo simbólico y con la psicosociología de la acción, respecto al colectivismo metodológico durkheimiano (Álvaro Estramiana et al. 2007b:10). Corbetta (1999:32-33), sostiene, además, que los conceptos de Verstehen de Weber y de Erlebnis, de Dilthey, o teoría de la empatía (Antiseri, 2005:62), han tenido una importancia relevante al indicar el trayecto que luego intentarán recorrer los sociólogos americanos del interaccionismo simbólico. Según la teoría de la empatía, el investigador tiene que ponerse en lugar del otro 
para entender cabalmente el fenómeno que quiere estudiar y explicar. Una técnica o perspectiva emic, fundamental según Blumer (2008:84) para tener en cuenta el punto de vista nativo (Kottak, 2011:59) y para que el sesgo del investigador no prevalezca. Una perspectiva que llevará a Cooley a la teoría del yo espejo (Caballero, 1997:2). Otros epistemólogos recientes, sin embargo, la rechazan porque la consideran poco científica o la tildan con la etiqueta de psicologismo. Entre ellos Aron, quien critica la validez epistemológica de la empatía como método científico (Di Nuoscio, 2004:176).

La definición de Corbetta y su referencia a Weber, aunque no podemos olvidar que Platt y Piovani (2011:256) niegan que la metodología de los chicagüenses se pueda explicar a partir del Versthen, se justifica por el hecho de que, a su juicio, en Weber se encuentran "todos los temas luego desarrollados por (...) la sociología fenomenológica, el interaccionismo simbólico y la etnometodología" (Corbetta, 1999:37). En realidad hoy tenemos claro que lo social y lo psicológico son dos caras de una misma realidad, más allá de las disputas entre psicología social sociológica y psicología social psicológica, y que los autores del interaccionismo tienen un papel destacado no sólo en la sociología propiamente dicha, sino también en la psicología social sociológica, aunque la versión psicológica de Allport prevaleció e impuso su sello en este campo de conocimiento (Álvaro Estramiana, 2007:14).

Más allá del origen más o menos weberiano, no cabe duda de que la tradición microsociológica tiene su origen en EE.UU., en respuesta y oposición a las grandes teorías, que allí empezaron a ponerse en duda con mucha anterioridad respecto a Europa y concretamente mucho antes que en países como Italia, Francia o, aunque en menor medida, España, donde las ideologías palingenéticas han seguido ejerciendo cierta influencia en el pensamiento hasta hace relativamente poco. Decimos "en menor medida", porque como recuerda, entre otros, Francisco Fernández Buey (1985:35), en España la producción marxista ha sido poco relevante desde el punto de vista teórico, respecto a Italia, Francia y Austria, con la excepción conocida de Sacristán, una producción menor respecto a otros vecinos europeos pero no ausente. Esa presencia ocultó durante mucho tiempo "la inflexión neoliberal (...) que estaba anidando en las universidades españolas" (Fernández Buey, 1991:134). En resumen, el interaccionismo simbólico y la microsociología se afirman también como respuesta y definitiva negación de "las concepciones globalizadoras, estructuralistas y objetivistas de la sociedad" (Caballero 1999:53; Blumer, 2008:92), y en oposición al modelo parsoniano de interacción humana (Blumer, 2008:101). Se mueven asimismo en contra de todas las verdades absolutas y de las certezas, y de las categorías y clases, separadas y enfrentadas, durante todo el siglo XIX y primera parte del XX, sin posibilidad de comunicación y de role-taking (Perrotta, 2005:61).

A lo largo del siglo XX la vida en sociedad se hace más compleja, las personas dejan de vivir en comunidades y comienzan a convivir en sociedades, dejan de tener una conciencia de clase tan bien definida, un proceso que concierne, entre otros, a los obreros de las grandes fábricas. Entra en crisis también el concepto de lucha de clases, algo que en realidad nunca tuvo una gran influencia en EE.UU. (Massari, 2004:68). Desde este punto de vista, hay que considerar como un elemento no casual el hecho de que el interaccionismo simbólico naciera en Chicago, "un espejo del liberalismo americano" (Picó y Serra, 2010:41), como atestigua también la ideología antirrevolucionaria del mismo Mead, y una ciudad en la que los sociólogos "no tenían que alejarse demasiado de sus despachos para encontrarse con su objeto de estudio" (Álvarez-Uría y Varela, 2004:286). No es casual, como decía, que el interaccionismo naciera en Chicago y no en una ciudad europea, como Turín, por poner un ejemplo, donde en los sesenta del siglo XX el pensamiento obrero tenía todavía mucho peso; una ciudad que Franco Sbarberi (1999:23) considera como el equivalente italiano de la París 
jacobina y de la Comuna, dado que en los sesenta se seguía tratando a Gramsci como un icono, y comunismo y antifascismo eran prácticamente percibidos como sinónimos (Furet, 1995:189). "Las clases sociales -dice Picó López (1997:96)- estuvieron siempre presentes en la sociología francesa e italiana". Y es imposible entender la sociología en Italia y en Francia, sobre todo la época de los años 50 y 60 del siglo XX, sin hacer referencia al marxismo (Picó López, 1997:106). Sólo unos pocos pioneros, como Ferrarotti en el campo de la sociología italiana, viajaron a EE.UU. para conocer de cerca esta nueva realidad en proceso de construcción.

En EE.UU., a finales de los cincuenta, Daniel Bell (1976) podía afirmar, obtorto collo, que se había constituido ya una sociedad de tipo post-industrial y podía empezar a reflexionar sobre la crisis de las ideologías. Confirma Alexander que EE.UU. fue el país en el que menos peso ha tenido la tradición marxista entre los intelectuales y donde en realidad "el radicalismo nunca cobró una forma manifiestamente colectivista" (Alexander, 2000:16).

El pragmatismo, acompañado por liberalismo, individualismo (Caballero, 1997:26-27), desarrollo industrial antes, y de la sociedad de los servicios después, constituye el contexto cultural, histórico, político y social que favorece el surgimiento y la difusión del interaccionismo en EE.UU. La importancia fundamental de este país desde este punto de vista es reconocida por un sociólogo crítico como Collins: "El microinteraccionismo no es la única tradición sociológica americana, pero en mi opinión representa la contribución más original que dio ese país al campo sociológico" (Collins, 1996:175). La forma de organizar el conocimiento y las nuevas ciencias sociales que se impusieron en EE.UU., basadas en estudios de alcance medio, acompañaron y en parte determinaron el ascenso de ese país como nueva fuerza hegemónica (Zunz, 2002:86-91), y determinaron a la vez el liderazgo de la sociología norteamericana sobre la europea, como ha denunciado Münch en sus análisis críticos (Picó López, 1997:100-101).

Desde el principio en la sociología estadounidense no hubo apenas, aparte de la relevante excepción parsoniana, sociólogos que se centraran en elaborar teorías holísticas y sistemas sociales, como sí hicieron en Europa en los albores de la disciplina Comte o Spencer, entre otros.

En los años treinta el interaccionismo simbólico, tras el gran desarrollo del principio, entra en crisis en EE.UU., (Álvaro Estraimiana y Garrido, 2007:23) por la progresiva afirmación de la escuela funcionalista, con una cosmovisión totalmente opuesta al interaccionismo, que volverá a recuperar importancia en el ámbito de la sociología estadounidense en los años sesenta, cuando empieza a debatirse la convicción de que, como dijo Kingley Davis en 1959 (Wallace y Wolf, 1985:27), cualquier reflexión sociológica científicamente válida tenía que ser al fin y al cabo funcionalista o inscribirse dentro del modelo estructural-funcionalista.

\section{3. ¿EXISTE UN CORPUS COMÚN ENTRE LOS AUTORES DEL INTERACCIONISMO? EL MANIFIESTO DE BLUMER COMO PUNTO DE PARTIDA}

La bibliografía especializada revela que tampoco existe consenso en torno a la verdadera identidad del padre y fundador de esta escuela, en general tan heterogénea y tan poco "escuela", en el sentido clásico que tiene esta palabra, en el lenguaje sociológico. De hecho, hay incluso quien niega que se pueda hablar de una verdadera escuela interaccionista, por la falta de una mínima koiné entre los autores, y también hay quien reconoce la necesidad de 
distinguir entre la escuela de Chicago y la de Iowa, es decir, fundamentalmente, entre Blumer y Kuhn (Caballero, 1999:53). Tampoco falta quien (Perrotta, 2005:24) reduce significativamente la envergadura del enfrentamiento entre seguidores de Blumer y de Kuhn, y atribuye a Reynolds la leyenda de estas dos facciones, "la una contra la otra, armadas". Es el caso de Denzin. Pons Díez (2010:30-31) diferencia tres corrientes dentro del interaccionismo: la estructuralista (representada fundamentalmente por Styker), la interaccional (con Turner), y la microinteraccionista (Goffman).

Muchos consideran a G.H. Mead como el verdadero padre del interaccionismo simbólico o, al menos, el principal precursor (Álvaro Estramiana et. al., 2007:14) pero en realidad Mead no era un sociólogo, sino un filósofo, admirador de la psicología de Watson, a cuyo modelo basado en la combinación de estímulo y respuesta añadió un pasaje intermedio, el de la interpretación (Blumer, 2008:114): es el llamado conductismo social, en contraposición al conductismo tradicional (Rizo García, 2011:78). Pero Lewis y Smith han subrayado la existencia de importantes diferencias entre el pensamiento de Mead y la reflexión sociológica de los otros autores del interaccionismo. Y, según Anselm Strauss, la influencia de Mead en el Departamento de Sociología de la Universidad de Chicago fue mucho más limitada de lo que se ha dicho después (Monti, 2008:47). Confirma a este respecto Alexander que el filósofo pragmatista Mead fue muy importante para la teoría sociológica moderna, pero en realidad "el interaccionismo contemporáneo se ha alejado del impulso institucional y colectivo de Mead" (Alexander, 2000: 175). Pons Díez considera a Mead como uno de los principales precursores del interaccionismo, igual de importante, pero no más, que Dewey (Pons Díez, 2010:25). En realidad ha sido Blumer quien ha construido la idea de Mead como padre del interaccionismo, dado que, prácticamente desde la primera página de su manifiesto interaccionista (Blumer, 2008:33), reconoce la deuda que tiene con su maestro Mead, capaz de "poner de verdad las bases del interaccionsimo simbólico". No obstante y según Esther Monti, Blumer no tuvo contactos directos con Mead, fue él quien convirtió a Mead en un sociólogo (Denzin, 2009:52).

Denzin (2009:54), más allá de los nombres propios, habla sin embargo de una fase canónica para el interaccionismo simbólico que se iniciaría en 1890. Otros empiezan a hablar del interaccionismo a partir de las reflexiones de otros filósofos pragmatistas como James, o de los análisis de Cooley y de los estudios de Thomas, con su fuerte dosis de psicología social dentro de la sociología (Picó y Serra, 2010:68).

Sí podemos afirmar, retomando a Rizo García (2011:81), que ha sido Blumer quien ha teorizado más exacta y claramente sobre los principios del interaccionismo simbólico. Blumer, a la vez discípulo y admirador de Mead (Rauty, 2008:13), fue el autor que acuñó e hizo célebre el concepto, a partir de 1937-1938. Y fue él quien llegó a la sociología casi por azar, pensando que sociología y socialismo fuesen sinónimos (Monti, 2008:36) y consiguió realmente hacer del interaccionismo simbólico "una fuerte corriente subterránea y una voz de oposición" (Collins, 1996:190). Además ejerció un papel fundamental en la definición del marco teórico y metodológico del interaccionismo.

El año 1937 representa un hito fundacional, justo un siglo después de que Comte revelara que ya era hora de sustituir la vieja física social por la sociología, una ciencia más moderna y positiva. Un siglo más tarde, Blumer, acuñando esta nueva expresión, implícitamente declaraba la necesidad de que la sociología dejara ya de ocuparse sólo de máximos sistemas y profundizara en las dificultades de las interacciones sociales en la vida cotidiana y de la comunicación interpersonal.

Podemos afirmar, pues, que fue Blumer quien acuñó el concepto de interaccionismo simbólico y que el interaccionsimo nació en EE.UU. en las primeras décadas del siglo XX. 
Estas pocas realidades son las únicas "casi certezas" que tenemos a nuestra disposición, porque, como afirma Rock, "la divisiones y la ausencia total de unidad caracterizan la Escuela de Chicago desde sus orígenes" (Rock, 1979:99). Ni siquiera la importancia fundamental de la Universidad de Chicago en el origen y en el desarrollo del interaccionismo es afirmada y apoyada por todos. Según Denzin (2009:64) no hay que caer en el error de utilizar interaccionismo y Escuela de Chicago como si fueran sinónimos, dado que dentro de la esta encontraríamos en realidad cuatro corrientes distintas, una de las cuales sería el propio interaccionismo. Y la Universidad de Chicago fue sólo una de las instituciones académicas que contribuyeron al desarrollo de esta corriente de pensamiento sociológico, a la par que Michigan, Iowa y Berkley (González Radio, 1995:24).

En el estado epistemológico actual, por ejemplo, no seríamos capaces de contestar a estas preguntas, entre otras: ¿Cuáles son, al fin y al cabo, las características fundamentales de los autores interaccionistas? El punto de unión de todos estos autores, por otra parte tan diferentes entre ellos, podría estar en la centralidad que tiene el concepto de interacción con los otros mediada a través de símbolos. ¿Pero es suficiente este elemento para que se pueda hablar de una escuela sociológica con un corpus común? En suma ¿hay un corpus común, y de verdad se puede hablar de escuela, o sería mejor hablar de escuela sin escuela, como en el caso de Palo Alto? ¿Fue Ervin Goffman un autor perteneciente a la escuela del interaccionismo simbólico, por el uso que hace el sociólogo de Manville de la teoría del "otro generalizado" de Mead (Straniero, 2004:42) o, como dice Collins y confirma Gonos (quien considera a Goffman directamente y sin duda alguna un autor estructuralista) sólo unos superficiales incompetentes pueden incluir a este autor tan genuinamente durkheimiano (Collins, 1996:201) y tan ideológicamente conservador en esta escuela? ¿Erving Goffman se puede considerar como un simple repetidor, poco original, del análisis social de Durkheim, un durkheimiano más durkheimiano que él (Burns, 1992:77)? ¿O se trata simplemente de un autor con intereses y enfoques variados y que analiza la vida cotidiana a partir de una perspectiva dramatúrgica que se puede considerar solamente muy cercana, y no equivalente, a la interaccionista (Carabaña y Lamo de Espinosa, 1978:176)? Tom Burns (1992:38), quien le conoció y trabajó con él, confirma esta idea: "Goffman no rechazaba el interaccionismo simbólico pero intentó mantenerse alejado del mismo". A propósito de la herencia durkheimiana, Burns (1992:44) la relaciona sobre todo con el concepto de "hecho social" y con el concepto durkheimiano de "sacralidad de la persona", que el mismo Goffman habría hecho menos generalizante y más concreto (Burns, 1992:61-62). En síntesis, Burns llega a describir, sin afirmarlo directamente, a un Goffman estructuralfuncionalista, no obsesionado como Parsons, pero sí interesado, en el problema de la homeostasis social. Y a la misma consideración llegan Herrera Gómez y Soriano Miras, que ponen en evidencia la influencia de Durkheim y de Freud en Goffman en mayor medida que la de Mead (Herrera Gómez y Soriano Miras, 2004:90), aunque a la vez afirman que Goffman concede más libertad al individuo para construirse un espacio autónomo dentro del control social respecto a Durkheim. El colectivismo ontológico y metodológico de Durkheim (Di Nuoscio, 2006:115) es mitigado y suavizado en Goffman. Diferente es la interpretación que da de la reflexión sociológica goffmaniana Rizo García, quien en lugar de poner en evidencia la herencia durkheimiana opta por dar mayor importancia a los estudios que Goffman realizó con Everett Hughes -sociólogo que trabajó en Chicago pero que no puede ser afiliado al interaccionismoy considera al fin y al cabo a Goffman como un autor interaccionista (Rizo García, 2011:81). Dada la diversidad de interpretaciones del pensamiento goffmaniano habría que seguir las 
pistas de Romania (2012:83) y diferenciar en fases y momentos el pensamiento y la producción sociológica de Goffman.

El interaccionismo se caracteriza también, como nos recuerda Blumer (2008:74), por privilegiar las investigaciones cualitativas sobre las estadísticas y los métodos cuantitativos, en auge en la época del dominio funcionalista (Caballero Romero, 1991:84) y utilizar sobre todo el estudio directo del mundo social empírico (Blumer, 2008:81) a través de la observación participante como técnica de investigación privilegiada, "para entender las personas dentro de su marco de referencia" (Pons Díez, 2010:30). Pero ni siquiera desde este punto de vista hay acuerdo. Platt, entre otros, considera más un mito que un realidad el abordaje cualitativo de la escuela de Chicago (Piovani, 2011:248), porque importantes cuantitativistas ejercieron un papel relevante en la misma (Picó y Serra, 2010:118), porque el número de tesis doctorales que se presentaron en el ámbito de ese departamento y que utilizaban los métodos cuantitativos superaba con creces el número de las que se servían de las técnicas cualitativas, en el período 1949-1962 (Picó y Serra, 2010:185); y por último también porque el mismo término "observación participante" fue aclarado y sistematizado sólo en 1955 (Picó y Serra, 2010:164). Los chicagüenses desde este punto de vista anticiparon la triangulación metodológica actual, utilizando a la vez estadísticas y técnicas cualitativas descriptivas (Picó y Serra, 2010:23).

Todas estas cuestiones acompañan el debate en torno al interaccionismo simbólico, y a su importancia teórica y práctica en el discurso teórico de la sociología moderna y contemporánea, y demuestran la dificultades que acompañan el estudio y la delimitación espaciotemporal de esta escuela.

En conclusión, aunque existen algunos principios aceptados por todos, es complicado, si no casi imposible, dar una definición y delimitar con exactitud en el tiempo el surgimiento del interaccionismo simbólico y esto demuestra la dificultad de llevar a cabo una depuración conceptual mediante la confrontación analítica de los estudios existentes. Como decía Blumer (2008:213) a propósito de la ambigüedad e imprecisión de los conceptos que se usaban en la psicología social, es difícil identificar "todo lo que tendría que entrar en el objetivo del concepto [de interaccionismo simbólico]" y diferenciarlo de las cosas que son externas al mismo. Si el significado conceptual es tan controvertido, más difícil será aún percibir cuál ha sido la influencia de esta corriente de pensamiento más allá de su tierra de origen, EE.UU.

\section{INTERACCIONISMO SIMBÓLICO Y LITERATURA: ¿PIRANDELLO Y GOZZANO COMO GOFFMAN?}

Una vez aclarado nuestro marco teórico y definido qué se entiende por interaccionismo simbólico, con todas las dificultades que acompañan una definición conceptual de este tipo, nos gustaría intentar contestar a una de las preguntas de investigación que nos habíamos propuesto al principio: ¿Es posible construir una nueva afinidad electiva entre autores de la literatura italiana y los autores del interaccionismo simbólico por las temáticas tratadas por ambos? González García (1989:15) justifica su trabajo sobre Weber y Kafka, argumentando que sólo desde un enfoque multidisciplinar se puede intentar desvelar "el misterio Kafka". Esta pregunta que nos hemos hecho y la consiguiente hipótesis que hemos formulado, se justifican por el hecho de que para entender el interaccionismo simbólico y los distintos misterios que lo acompañan necesitamos un enfoque parecido al que utilizó González García. 
Como hipótesis, sobre la base de nuestro conocimiento previo y de nuestra cultura de referencia, partimos del análisis de Pirandello y Gozzano, que a nuestro juicio son los autores que más podrían acercarse a las temáticas tratadas. Efectivamente, a primera vista, en el caso italiano, que es el que mejor conocemos, el interés por las pequeñas cosas, por los significados que atribuimos a la realidad cotidiana y a la identidad cambiante de las personas, ha tenido cierta relevancia también en el mundo literario y en la poesía, que desde este punto de vista han precedido y se han anticipado a la reflexión sociológica, lo cual no representaría una novedad, dado que en otros momentos históricos la literatura ha anticipado y precedido a la sociología en el análisis de los problemas y cuestiones sociales (Picó y Serra, 2010:78). Pero, según Jacinto Choza, no sólo Pirandello y la literatura italiana pueden sugerir estas analogías. Junto a Pirandello, tendríamos que citar a Heidegger, Freud, Wittgenstein, Joyce, Kafka, Unamuno, Rilke, Pessoa; porque todos ellos responden "a un arte máximamente reflexivo y crítico, que indaga muy radicalmente en la existencia humana" (Choza, 1999:43). Pero para delimitar nuestro campo de investigación y no convertir lo mismo en algo inabarcable, nos hemos limitado a formular e intentar contestar a la siguiente pregunta: ¿Fueron interaccionistas Pirandello y Gozzano, por citar a dos figuras consagradas de la literatura italiana? Rosalba Perrotta contesta decididamente que sí. En realidad, según lo que hemos podido detectar con la bibliografía a nuestro alcance, Pirandello no llegó a leer directamente a los autores del interaccionismo, dado que esta escuela sociológica estaba todavía en fase de construcción por aquel entonces. Sin embargo, sí que recibió la influencia de Alfred Binet sobre los cambios de personalidad (Misan Montefiore, 2007:394).

Por lo que concierne a Gozzano, el otro autor que habíamos nombrado en nuestra hipótesis, es, según Benedetto Croce, el único poeta italiano verdadero del siglo XX (Mengaldo, 1978:90). Es un autor con una fuerte implicación psicológica y sociológica, que, por lo que hemos podido averiguar, tampoco llegó a conocer o a leer directamente a Mead, Blumer o Cooley, pero que redescubrió, al igual que estos sociólogos, las pequeñas cosas, la vida cotidiana y la importancia del proceso de significación de las cosas, de esos procesos a los que los interaccionistas han dado mucho peso. Como el Goffman interaccionista, Gozzano utiliza, por ejemplo, palabras nuevas para referirse a conceptos ya conocidos, porque tiene en cuenta en sus definiciones el punto de vista de los observados, y no sólo el suyo, privilegiado, de observador. Así queda patente cuando habla de civettare o de crestaia (Mengaldo, 1978:93). Palabras inventadas por Gozzano difícilmente traducibles al español (civettare se refiere a mujeres burguesas que intentan llamar la atención y crestaia se usa para mujeres de clase media que aman estar a la moda). La mujer descrita por Gozzano es simple, poco agraciada, real, no es la mujer de D'Annunzio, Ermione, o la Beatriz de Dante (Marangon, 2012:103). Gozzano contrapone a las grandes puestas en escena, espectaculares, de D'Annunzio, las cosas pobres. En Gozzano "la lengua de la tradición poética de D'Annunzio baja hasta incluir el lenguaje de la banalidad, de lo cotidiano" (Marangon, 2012:99) y en él se rompe la división entre mundo culto y profano, entre palabras literarias y no literarias, anticipándose no sólo a los interaccionistas sino también a los antropólogos culturales que se oponen a la idea de una alta cultura que se distinguiría de la popular -o incultura- como si de una sustancia que tenemos dentro de nuestros cuerpos se tratara. Todo es cultura, incluso las realidades insignificantes del mundo cotidiano (Kottak, 2011:34); en esto Gozzano anticipa a Goffman, pero no a Mead, dado que Goffman había dado voz a los internados, en lugar de dedicarse a las grandes teorías o a elaborar un programa más de revolución para la humanidad. Pero a la vez anticipa la sociología de la vida cotidiana y la fenomenología de Schültz y por consiguiente no puede ser considerado como elemento 
suficiente para llevar a cabo un paralelismo empíricamente sostenible (Álvaro Estramiana, et al., 2007b:87-88). También Garfinkel presta atención a los hechos de la vida diaria, algo que tiene en común toda la microsociología. Con el interaccionismo y la microsociología que se interesa por la vida cotidiana, se rompe, si queremos, también la distinción académica entre antropología y sociología; la sociología, como la antropología cultural, empieza a observar de cerca esas realidades, a veces reducidas, que quiere estudiar (Kottak, 2011:63).

Un paralelismo análogo se puede realizar, como hemos venido diciendo, entre Goffman y Pirandello. En Goffman la herencia durkheimiana y el colectivismo metodológico y ontológico, sin embargo, retomados directamente del pensador francés, impiden que se pueda hablar de rechazo absoluto y total de las macroteorías y de las filosofias holísticas, que sí se encuentra en Pirandello, quien en una carta a su hijo denuncia la incapacidad de la filosofía para solucionar los problemas humanos (Catalano, 1991:13). Pero en ambos la vida real, incluso la cotidiana, cuenta más que los conceptos abstractos y alejados de la realidad.

Pirandello, además, al igual que Goffman y su enfoque dramatúrgico, juega en sus obras con los infinitos selves que un individuo puede adoptar y mostrar durante la interacción. En Uno, nessuno, centomila el protagonista, Vitangelo Moscarda, se encierra en una residencia para indigentes y vive entre mendigos, tal como Goffman hizo entre los enfermos para llevar a cabo su estudio sobre los hospitales psiquiátricos (Giudice y Bruni, 1973:253). Pirandello como Goffman juega en sus obras con el tema de las máscaras. Dice Baldovino, uno de sus personajes, "yo cuando me pongo aquí ante usted me convierto en lo que tengo que ser" (Misan Montefiore, 2007:402). Los personajes que protagonizan las novelas de Pirandello son por lo general indefinidos, cambiantes, sin una identidad precisa, exacta, siempre iguales, como ocurría en la literatura de los tiempos de Don Quijote o de Madame Bovary (Perrotta, 2008: 125), aunque, como nos ha revelado Choza, Pirandello amaba también a Cervantes y al Quijote (Choza, 1999:48). Según Rizo García (2011:82), en Pirandello, al igual que en Goffman -y en esto está el probable punto de encuentro entre los dos, que no se conocieron ni se leyeron- "persona" vuelve a significar máscara, prósopon, como en la Antigua Grecia. Según otros críticos literarios, como Misan Montefiore (2007:403), la literatura de Pirandello es más psicología que literatura propiamente dicha. Pero tiene también una clara implicación sociológica, como se hace evidente en Il fu Mattia Pascal con el protagonista que sufre las normas de convivencia social que obligan a una persona a tener un nombre y una identidad estable y reconocible (Giudice y Bruni, 1973:251). En Mead y Goffman, como en Pirandello, "los sujetos se forman bajo esta obligación de ser presentables" (Manero Guillamón, 2012:316); lo social y lo psicológico figuran como dos caras de una misma realidad.

Pirandello se acerca a la escuela interaccionista también porque, al igual que Thomas con su conocido teorema, considera y describe en sus novelas lo imaginario como más real que lo real, por sus consecuencias y por su potencia infinita (Misan Montefiore, 2007:401). "La realidad -dice Pirandello- no nos ha sido dada, la tenemos que hacer nosotros" (Battaglia, 1963:267). Hay personajes protagonistas de las novelas de Pirandello que no se limitan a interpretar papeles, sino que pierden de vista la diferenciación entre realidad y ficción, como atestigua esa actriz que llega a desmayarse por la intensidad de la identificación con su personaje (Choza, 1999:44). O como en el caso del profesor Lamis, protagonista de L'eresia catara, que no se da cuenta de que los alumnos de su clase en realidad no son personas sino chubasqueros sin cuerpos que se están secando (Giudice y Bruni, 1973:254). Las distinciones entre lo real y la ficción, la locura y la normalidad, la sociedad de los normales y la sociedad de los anormales o desviados (Catalano, 1991:35), el público y los espectadores... se anulan en Pirandello (Giudice y Bruni, 1973:257). En el teatro pirandelliano un fantasma puede llegar a tener "la misma consistencia que la realidad", según palabras de un protagonista de 
Cosí é se vi pare (Catalano, 1991:27). En ambos, Pirandello y Goffman, la experiencia biográfica tiene que estar relacionada con la producción intelectual. Pirandello, al igual que Goffman, tenía una mujer enferma mental.

Y las características en común no terminan: ambos fueron criticados por muchos de sus contemporáneos, acusados de ser poco serios y de querer sorprender a toda costa. Pirandello fue cuestionado por Croce, entre otros (Giudice y Bruni, 1973:259). Y Pirandello, al igual que Goffman, describe a los que sufren, a los vencidos, sin piedad humana, no como pobres víctimas, sino incluso poniendo en envidencia los aspectos más ridículos de los mismos, lo cual provocó las quejas de los críticos literarios y pensadores marxistas como Petronio que, sin embargo, comentaron positivamente las obras de Verga.

Goffman y los demás interaccionistas, como Pirandello, han sido criticados por sociólogos marxistas por no haber tenido en cuenta las desigualdades y haber jugado un papel conservador (Romania, 2012:74), a la par que por sociólogos estructuralfuncionalistas. Según sus críticos, Pirandello y Goffman se habrían puesto al servicio del poder.

\section{EL INTERACCIONISMO EN LAS REVISTAS ITALIANAS Y ESPAÑOLAS: ¿PRESENCIA O AUSENCIA?}

Como anticipamos en la introducción, hemos tomado en consideración y revisado las principales revistas sociológicas italianas y españolas para averiguar qué espacio dan al interaccionismo simbólico.

Por lo que atañe a las revistas italianas que hemos tomado en consideración estos son los resultados de nuestra investigación. En la revista de la editorial Franco Angeli, Sociologia e Ricerca Sociale, el interaccionismo es objeto de un número casi monográfico, el 90 de 2009. De hecho, en este número, la expresión "interaccionismo simbólico" aparece explícitamente citada en el título de un artículo escrito por el sociólogo Raffale Rauty: "L’interazionismo simbolico: un percorso di studio", de sólo tres páginas, que sirve como introducción a la misma. Luego vuelve a aparecer en un texto de Norman Denzin, "L'ereditá interazionista", un artículo mucho más extenso, que ocupa 27 páginas. A continuación va un artículo de Gianni Losito, dedicado a la interacción social, de 14 páginas. En otra revista, Sociologia della Comunicazione, se encuentran tres artículos relacionados, al menos en el título, con la Escuela del interaccionismo simbólico, lo cual demuestra la interdisciplinariedad de esta corriente que se ha ocupado de interacción, de símbolos, de lenguaje, de comunicación verbal y no verbal, de identidad y desviación, entre otros temas. En uno de estos tres artículos, en "Etnometodologia e scienze sociali", de Aurelia Marcarino ( $\left.n^{\circ} 19,1993\right)$, en realidad el tema principal es el análisis de la etnometodología, corriente microsociológica cercana al interaccionismo. En los otros dos (Capucci, Pier Luigi, "Infrastrutture del simbolico", no 38, 2005; Bartoletti, Roberta, "Oggetti dell'indifferenza. Simbolico del sociale e oggetti mediadi", $\left.n^{\circ} 35,2004\right)$ se habla más explícitamente de interaccionismo. Hay que recordar, además, que la editorial Il Mulino lleva años publicando una revista llamada Etnografia e Ricerca Qualitativa, que se dedica a hacer públicos los últimos resultados de las investigaciones cualitativas y de la observación participante en el campo internacional. Se trata de una revista que no se titula Symbolic Interaction como la conocida revista americana, pero que puede quedar inscrita dentro de esta corriente de investigación y análisis. Sin embargo, en esta publicación, más dedicada a los estudios de campo que a la investigación teórica, no hay ningún artículo sobre el interaccionismo simbólico. En las cabeceras de la 
editorial "Il Mulino" (Rassegna italiana di sociologia, Etnografia e Ricerca qualitativa, etc.) se encuentran cinco artículos dedicados a Goffman, pero ninguno directamente dedicado a la escuela del interaccionismo simbólico.

Entre las revistas españolas, según el buscador rápido de Dialnet (consultado el 30 de junio de 2014), se encuentran 308 documentos, de los cuales 233 son artículos relacionados de alguna forma con el tema del interaccionismo. Lo cual desmiente en parte nuestra hipótesis de un escaso interés hacia el interaccionismo en España. Varios artículos se ocupan de otros temas y sólo citan a autores del interaccionismo, por lo que se pueden relacionar muy a la lejana con esta escuela sociológica. Hay textos que son simples investigaciones cualitativas o que mencionan a algún autor interaccionista.

Además, en las principales revistas sociológicas las cosas cambian decididamente. En la Revista Española de Investigaciones Sociológicas (REIS), una de las principales, la expresión "interaccionismo simbólico" aparece explícitamente citada en el título de un solo artículo. Se trata de un número muy reducido, si tenemos en consideración que es una revista que se publica desde 1978 y que, por tanto, ha editado centenares de textos a lo largo de su historia. Pero resulta significativo que este texto, en el que se habla explícitamente de interaccionismo ya desde el título, aparece en el histórico número 1 de 1978. Se trata efectivamente de un artículo escrito por dos reconocidos sociólogos españoles, Julio Carabaña y Emilio Lamo de Espinosa, y que tiene además una extensión muy considerable (44 páginas). ¿Por qué es tan significativo este artículo, desde el punto de vista sesgado de nuestros objetivos investigadores? Sobre todo porque reconoce implícitamente lo que acabamos de decir, que en España, hasta entonces, el interaccionismo simbólico había recibido poquísima atención: "Pueden contarse con los dedos de la mano -dicen Carabaña y Lamo de Espinosa (1978:159)- los escritos en castellano sobre el interaccionismo simbólico". Podemos proponer un esbozo de explicación a este respecto: España en 1978 acababa de salir de una dictadura conservadora y clerical, y el mundo político y académico se dividía por lo general entre una derecha en proceso de democratización y una izquierda que se había radicalizado por su oposición al régimen (el PSOE en 1978 todavía se declaraba marxista y no había pasado por su Bad Godesberg). "Una polarización entre un funcionalismo ligado al empirismo americano y el marxismo influido por la teoría crítica europea" (Noya, 2004:166). Como recuerda Picó López (1997:104), la política anticomunista en Europa se jugó también en el campo sociológico, a través de fundaciones e inversiones económicas que tenían el objetivo de romper la contraposición derechas-izquierdas. Los autores del interaccionismo son por definición aideológicos o denotan cierta cercanía al liberalismo democrático (Denzin, 2009:53), una ideología política que ha tardado en imponerse en España e Italia, a pesar del esfuerzo estadounidense. La microsociología, además, ha sido considerada por lo general menos importante que la macrosociología (Lozano Maneiro, 2003:58) y este factor también ha tenido un cierto peso en la poca atención que se ha prestado al interaccionismo. Algo confirmado también por Javier Noya (2004:169), que recuerda la escasa atención que se ha dedicado a la teoría microsociológica en el ámbito español, aunque a la vez reconoce que en los años 80 el interaccionismo simbólico fue la corriente microsociológica que más espacio tuvo en España.

En realidad no sería correcto decir que en la REIS este el único artículo de teoría sociológica dedicado al interaccionismo $\mathrm{y}$, como anteriormente hemos anunciado, profundizando un poco más dentro de la misma revista, son varios y repetidos los artículos que más o menos directamente hablan de temas afines a nuestra investigación. Goffman tiene dos artículos dedicados a desarrollar su doctrina o a explicar su pensamiento, así como Mead, uno de los padres de la escuela, citado directamente en el título de dos artículos, en el número 
74 de 1996 y en el número 55 de 1991. En Política y Sociedad, directamente citado en el título no se encuentra ningún artículo dedicado al tema. En Sociedad y Utopía se localizan dos textos centrados en el interaccionismo ya desde el mismo título. En el primero, González Radio (1995) vuelve a presentar las pocas "casi certezas" que tenemos sobre el interaccionismo como escuela. En el segundo, Caballero (1997:37-39) explica en qué se diferencia el interaccionismo de Mead de la teoría del conflicto, del estructuralismo, de la teoría del intercambio, etc. A continuación presentamos una tabla que resume el espacio ocupado por el interaccionismo en algunas de las más significativas revistas italianas y españolas:

Tabla 1: El interaccionismo simbólico en revistas italianas y españolas. Número de artículos y páginas

\begin{tabular}{|l|l|l|}
\hline ITALIA & $\mathrm{N}^{\circ}$ de artículos & $\mathrm{N}^{\circ}$ de páginas \\
\hline Sociologia e Ricerca Sociale & 3 & $3+27+14$ \\
\hline
\end{tabular}

\begin{tabular}{|l|l|l|}
\hline ESPAÑA & $\mathrm{N}^{\circ}$ de artículos & $\mathrm{N}^{\circ}$ de páginas \\
\hline REIS & 1 & 44 \\
\hline Sociedad y Utopía & 2 & $16+19$ \\
\hline
\end{tabular}

Fuente: elaboración propia.

\section{LOS MANUALES SOBRE EL INTERACCIONISMO SIMBÓLICO Y LAS INVESTIGACIONES CON EL MÉTODO DE BLUMER Y GOFFMAN EN ITALIA Y EN ESPAÑA}

En Italia, por lo que hemos podido averiguar, hay más investigaciones publicadas sobre Goffman que manuales sobre el interaccionismo simbólico como escuela. El libro de Esther Monti (2008) a nuestro juicio no puede ser considerado como un manual completo y exhaustivo, porque presenta de forma breve y esquemática la vida de algunos de los sociólogos interaccionistas, pero no describe los orígenes de la escuela y la koiné presunta o real entre los autores. Monti lleva a cabo una elección personal de los autores más influyentes dentro de esta corriente: Blumer, Strauss, Turner, Shibutani y Goffman. Pero no dedica ninguna reflexión sobre otros autores igual de relevantes, como Theodore Sarbine, que Romania (2012:53) considera de gran influencia dentro de la teoría interaccionista de los roles. Picó y Serra (2010:XVII) recuerdan los estudios de Tomasi y de Caccamo, pero se trata de manuales sobre la escuela de Chicago y no específicos sobre el interaccionismo simbólico.

Según hemos podido comprobar, al menos tres autores actuales pueden inscribirse dentro de este enfoque en el ámbito de la sociología italiana contemporánea, aunque ninguno de ellos ha publicado manuales exhaustivos y completos sobre los orígenes y autores pertenecientes al interaccionismo. Se trataría de Vincenzo Romania, Esther Monti y Rosalba Perrotta, que han publicado libros sobre temáticas propias del interaccionismo o han aplicado en sus investigaciones técnicas cualitativas típicas del mismo, como la observación participante, para estudiar cuestiones como la marginalidad y la inmigración, entre otras. De Perrotta recordamos Cuore di farfalla (2010), un estudio cualitativo sobre los discapacitados, además de Cornici, specchi e maschere (2005). De Vincenzo Romania hay que destacar Farsi passare per italiani (2004), un estudio sobre el mimetismo social y las máscaras que 
utilizan los inmigrantes para huir de las discriminaciones. Y también Ruoli, identitá, interazioni en el que el mismo autor italiano confirma cuanto hemos venido diciendo hasta aquí: "En Europa (...) hay una general amnesia (...) hacia el interaccionismo simbólico" (Romania, 2012:1). Este autor afirma también que el número total de sociólogos italianos que se consideran interaccionistas no supera la decena (Romania, 2012:3). Casi absoluto es el desconocimiento que hay en la teoría sociológica italiana de un interaccionista como Ralph Turner, pensador que, según Romania (2012:67), tiene un papel relevante; lo que justificaría indirectamente también la decisión de Esther Monti de presentar como central la figura de Turner en el interaccionismo sociológico americano. Existe otra investigación sobre los presos detenidos en la cárcel de Rebibbia, Oltre l'istituzione totale (Esposito y Turco, 2011) que retoma claramente en el título y en la metodología investigadora el estudio goffmaniano sobre los internados. Por último cabe destacar que el manual quizá más valioso que existe sobre el interaccionismo publicado por Franco Angeli y obra de Meltzer, Petra y Reynolds (1980), es decir, por autores no italianos, y que es la traducción de una obra en lengua inglesa, hoy está descatalogado.

En España en 2010 se ha publicado la obra de Picó y Serra sobre la Escuela de Chicago, un texto que reconstruye el ambiente histórico y social que hizo de marco al surgimiento de esta escuela. Pero Picó y Serra dedican pocas páginas al interaccionismo simbólico en sí y se centran más en la ecología urbana de Park que en Mead o Goffman.

El catedrático de Sociología Octavio Uña publicó en diciembre de 2009, en Nuevos ensayos de sociología y comunicación, unas interesantes reflexiones sobre el interaccionismo simbólico y el papel fundamental que ejerció Blumer, pero tampoco podemos considerarlo como un manual, en el sentido clásico del término, sobre esta materia.

En suma, no hay ningún estudio que se centre sólo en el interaccionismo simbólico, al menos por lo que hemos podido averiguar con nuestra investigación. El libro coordinado por Álvaro Estramiana y Garrido Luque (2007b:22) podría ser considerado casi un manual sobre el tema, dado que se centra en la psicología social sociológica y reconoce desde el principio que el enfoque más significativo dentro de este campo de conocimiento es el interaccionista, aunque, en realidad, no son muchas las páginas al respecto, considerando que se trata de un volumen demasiado sintético desde este punto de vista. Sin embargo, sí que se encuentran capítulos dedicados al tema en varios manuales de teoría sociológica. Podemos afirmar que ni siquiera la figura de Goffman en España "ha tenido la penetración que hubiera sido deseable" (Lozano Maneiro, 2003:57). Además, también en España, se han publicado investigaciones cualitativas que toman como referencia las aportaciones de los interaccionistas. Por ejemplo, se puede citar la que realizada por Fernando Esteban, Ramón Alós y otros (2014), que estudiaron a través de una metodología explicativa mixta la reinserción laboral de los ex detenidos, tomando en consideración, entre otras, la teoría de las etiquetas sociales y de los estigmas.

Frente a este escaso interés en España e Italia, en el ámbito internacional se han publicado en los últimos años "muchos manuales sobre el interaccionismo y la psicología social sociológica" (Álvaro Estramiana y Garrido Luque, 2007b:118).

Por último, a través de la aplicación books.google.com/ngrams, hemos comprobado que la parábola del interaccionismo simbólico es parecida en los libros editados en lengua española y los que se han publicado en lengua italiana y que pertenecen al amplio archivo de libros de Google. En la década 1960-1970 los puntos más bajos en español son 1965 y 1967 y, a partir de 1970, hay un clara inversión de tendencia y un notable crecimiento. En lengua italiana hasta 1964 no hay ningún registro sobre el interaccionismo simbólico. Al igual que ocurre en español, aunque con más altibajos, el gráfico se dispara hacia arriba a partir de 1990. En 
Italia son todavía pocos los editores que han utilizado esta opción de digitalización parcial de los textos (Mazzucchi, 2008:131).

Tabla 2.1: La presencia del interaccionismo simbólico en los textos de lengua italiana de 1970 a 2000 según books.google.com/ngrams

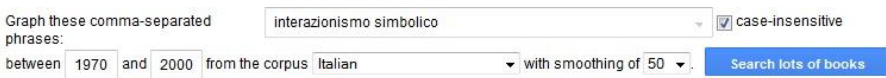
between 1970 and 2000 from the corpus Italian with smoothing of 50

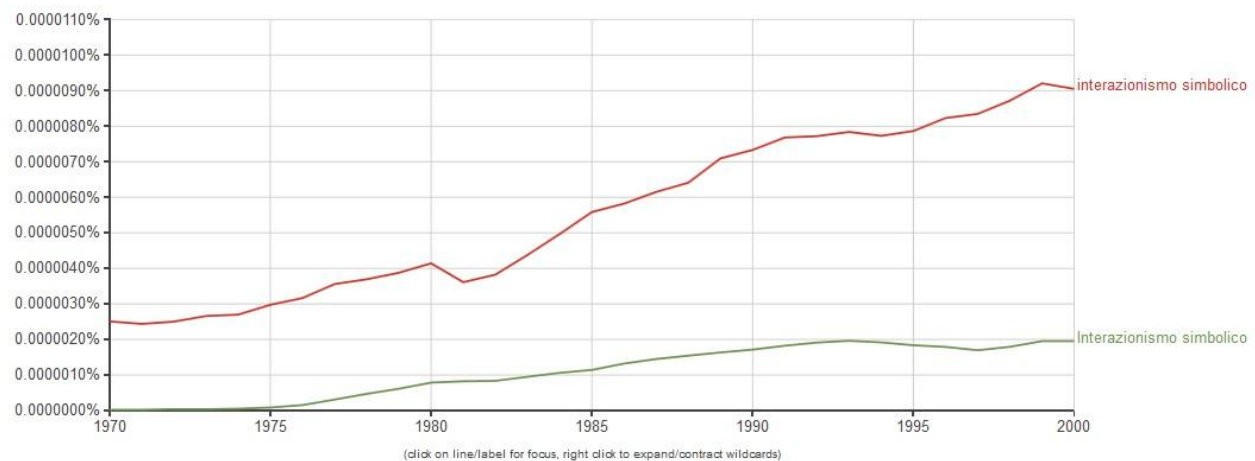

Search in Google Books:

$\underline{1970-1975} \underline{1976-1996} \quad \underline{1997} \underline{1998-1999} \quad \underline{2000} \quad$ interazionismo simbolico $\quad$ Italian

Tabla 2.2: La presencia del interaccionismo simbólico en los textos de lengua española desde 1970 a 2000 según books.google.com/ngrams.

Graph these comma-separated
phrases:
between 1970 and 2000 from the corpus Spanish

between 1970 and 2000 from the corpus Spanish with smoothing of 8 Search lots of book:

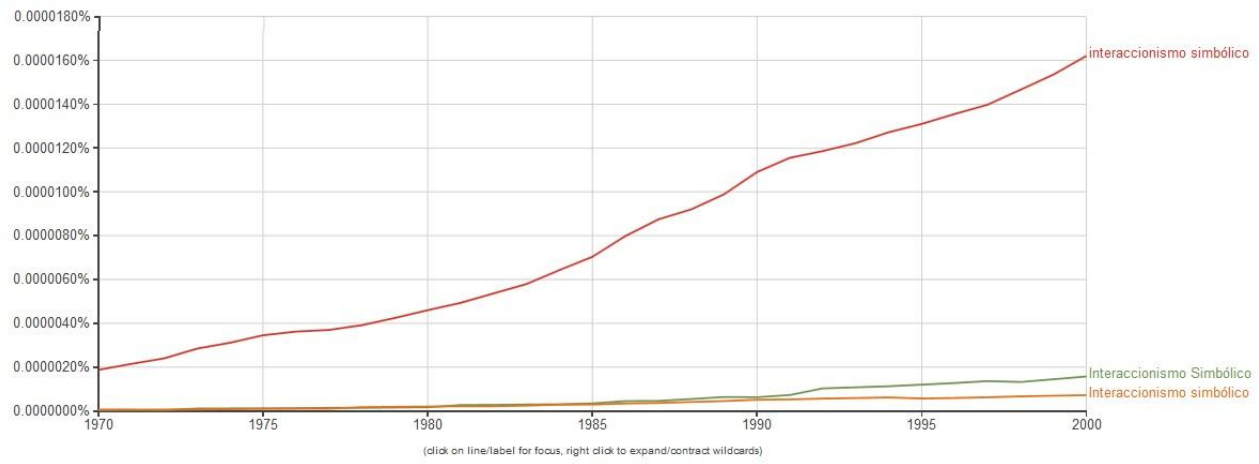




\section{RESULTADOS Y CONCLUSIONES}

Los resultados que hemos extraído de nuestra investigación son varios y contradictorios:

1. Cuando se estudia el interaccionismo simbólico y a pesar de los intentos producidos, la delimitación conceptual es muy controvertida, como de hecho ocurre a menudo en el ámbito de las ciencias sociales.

2. Nuestra evaluación nos hace juzgar que no se ha explorado cabalmente el interaccionismo simbólico como gran escuela sociológica, a la par que el funcionalismo y la teoría del conflicto. Es, por lo tanto, posible decir que, de acuerdo con las fuentes analizadas, al menos en Italia y en España, el interaccionismo necesitaría más espacio en publicaciones y revistas, lo que da la razón a Picó y Serra (2010: XVI) cuando aseguran que "a medida que nos acercamos al Mediterráneo el panorama se ensombrece". En el mundo anglosajón, por las razones que hemos descrito en parte, ha habido mucho más interés hacia esta corriente sociológica. Y desde este punto de vista, el artículo publicado en España en 1978 por Lamo de Espinosa y Julio Carabaña representa quizá un hito que ha abierto un camino todavía por explorar. Aunque en los últimos tiempos han aumentado considerablemente las investigaciones sobre la escuela interaccionista o las que utilizan métodos, que podríamos llamar interaccionistas, como la observación participante, pero pocas de estas se fundamentan en teorías microsociológicas como el interaccionismo. En realidad siguen siendo limitados los estudios teóricos sobre el interaccionismo simbólico que se centren en su desarrollo, su origen, sus limitaciones, en síntesis, su delimitación conceptual. Y este aspecto es confirmado prácticamente por todos los que se han dedicado al tema. Estas consideraciones habría que encuadrarlas en el ámbito de la tendencia más general de la sociología española, pero se trata de un trabajo que remitimos a futuras investigaciones.

3. Es importante señalar que se trata de una corriente o escuela interdisciplinar. Se habla del interaccionismo incluso en una revista médica donde el tema de discusión son los pacientes obesos (Ocampo Barrio y Pérez Mejía, 2010). El interaccionismo tiene cierto espacio también en revistas de educación. En la revista EduPsykhé (Pons Diez, 2010) Pons Díez habla de las aportaciones del interaccionismo en la psicología social. Pero ni siquiera en el campo de la psicología el estudio del interaccionismo y su presencia en manuales y revistas puede ser considerada signficativa (Pons Díez, 2010: 24). La misma psicología social sociológica, por lo general, ha tenido hasta el momento poco espacio en España, respecto a la psicología social psicología (Álvaro Estramiana, Garrido Luque [et al.], 2007b: 14).

4. Nos hemos centrado en a analizar la literatura crítica y las fuentes secundarias a nuestro alcance sobre Gozzano y Pirandello y hemos abordado directamente sólo una parte de sus textos. En el estado epistemológico actual la hipótesis de que se pueda construir una nueva afinidad electiva no puede ser ni refutada ni confirmada. Podemos decir que Pirandello estudió los textos de Alfred Bidet, y, más que literatura, la suya es una reflexión de carácter psicológico, pero esto no es suficiente para poder contestar afirmativamente a la pregunta de la investigación. Sí podemos afirmar que no hay una influencia directa de la sociología interaccionista en Pirandello y Gozzano, y hemos llevado a cabo un paralelismo o una afinidad construida desde la contingencia. Tampoco se pueden obviar las diferencias que transcurren entre la Sicilia de Pirandello y los EE.UU. de Goffman, dos espacios geográficos y sociales que tienen poco en común. Dejamos por consiguiente para futuras investigaciones un desarrollo más amplio sobre las posibles relaciones entre los movimientos literarios y artísticos que tratan temas propios del interaccionismo y esta corriente sociológica, para poder demostrar así, de forma más contundente, que las posibles y nuevas afinidades 
electivas con Gozzano, Pirandello y los llamados crepuscolari tienen cierto fundamento teórico y consistencia epistemológica.

\section{BIBLIOGRAFÍA}

ALEXANDER, J. (2000). Las teorías sociológicas desde la Segunda Guerra Mundial. Barcelona: Gedisa.

ÁLVAREZ-URÍA, F. y VARELA, J. (2004). Sociología, capitalismo y democracia. Madrid: Morata.

ÁlVARO ESTRAMIANA, J. L. y GARRIDO LUQUE, A. (2007). "Orígenes psicológicos de la psicología social", en Reis, 118: 11-26.

ÁLVARO ESTRAMIANA, J. L.; GARRIDO LUQUE, A. et al. (2007b). Introducción a la psicología social sociológica. Barcelona: Editorial UOC.

BARTOLETTI, R. (2004). "Oggetti dell'indifferenza", en Sociologia della Comunicazione, 35: 43-60.

BATTAGLIA, S. (1963). "La condizione umana nell'arte di Pirandello". En A. Giudice y G. Bruni, G. (1973). Problemi e scrittori della letteratura italiana. Torino: Paravia.

BELL, D. (1976). El advenimiento de la sociedad postindustrial. Madrid: Alianza.

BLUMER, H. (2008). Interazionismo simbolico. Bologna: Il Mulino.

BURNS, T. (1992). Erving Goffman. Bologna: Il Mulino.

CABALLERO ROMERO, J. J. (1991). "Etnometodología: una explicación de la construcción social de la realidad", en Reis, 56: 83-114.

CAPUCCI, P. L. (2005). "Infrastrutture del simbolico", en Rivista Sociologica della Comunicazione, 38: 41-57.

CARABAÑA, J. y LAMO DE ESPINOSA, E. (1978). "La Teoría social del Interaccionismo simbólico. Análisis y valoración crítica", en Reis, 1: 159-203.

CATALANO, E. (1991). La maschera dimenticata. Pirandello e il plurale del teatro. Fasano: Schena Editore.

CHOZA, J. (1999). "Las representaciones del yo en Pirandello", en Concepciones y narrativas del yo. Thémata, 22: 41-57.

COLLINS, R. (1996). Quattro tradizioni sociologiche. Bologna: Zanichelli.

- (2008). L'intelligenza sociologica. Caserta: Ipermdium Libri.

CORBETTA, P. L. (1999). Metodologia e Tecniche della Ricerca Sociale. Bologna: Il Mulino.

DE CASTRIS, L. (1967). "Le condizioni del personaggio nel teatro di Pirandello" en A. Giudice y G. Bruni (1973). Problemi e scrittori della letteratura italiana. Torino: Paravia.

DE NARDIS, F. (2011). Sociologia comparata. Appunti sulle strutture logiche della ricerca sociopolitica. Milano: Franco Angeli.

DENZIN, N. (2009). "L'ereditá interazionista", en Sociologia e Ricerca sociale, 90: 48-74.

DI NUOSCIO, E. (2004). Tucidide come Einstein. Catanzaro: Rubbettino.

- (2006). Il mestiere dello scienziato sociale. Napoli: Liguori.

ESTEBAN, F. y ALÓS, R. et al. (2014). "La inserción laboral de ex reclusos. Una aproximación cualitativa", en REIS, 145: 181-204.

FERNÁNDEZ BUEY, F. (1985). "Marxismo en España", en Sistema, 66: 25-43.

- (1991). "Veinte años de marxismo en España", en Sistema, 100: 129-142.

FURET, F. (1995). Il passato di un 'illusione. Milano: Mondadori.

GIUDICE, A. y BRUNI, G. (1973). Problemi e scrittori della letteratura italiana. Vol.III. Torino: Paravia.

GOFFMAN, E. (2008). Internados. Buenos Aires: Amorrortu.

GONZÁLEZ GARCÍA, J. M. (1989). La máquina burocrática. Afinidades electivas entre Max Weber y Kafka. Madrid: Visor.

GONZÁLEZ RADIO, V. (1995). “El interaccionismo simbólico”, en Sociedad y Utopía, 6: 59-76.

HERRERA GÓMEZ, M. y SORIANO MIRAS, R. M. (2004). "Ervin Goffman y la fragmentación de la vida cotidiana", en Sistema, 181: 55-91. 
KOTTAK, C. P. (2011). Antropología cultural. México: McGrawHill.

LOSITO, G. (2008). "Interazione sociale e interazione con i testi mediali", en Sociologia e Ricerca sociale, 90: 20-33.

LOZANO MANEIRO, B. (2003). "En el aniversario de Ervin Goffman (1922-1982)”, en Reis, 102: 4761.

MARRERO-GUILLAMÓN, I. (2012). "Descentrar el sujeto. Ervin Goffman y la teorización del sujeto", en Revista Internacional de Sociología, vol. 70, 2: 311-326.

MARANGON, G. (2012). "Guido Gozzano: dal Decadentismo al cammino moderno della poesia italiana", en Revista de Filosofía canónica, vol. 29, 1: 97-109.

MARCARINO, A. (1993). "Etnometodologia e Scienze Sociali", en Sociologia della Comunicazione, $\mathrm{n}^{\mathrm{o}} 19$, s.p.

MASSARI, O. (2004). I partiti politici nelle democrazie contemporanee. Roma-Bari: Laterza.

MAZZUCCHI, E. (2008). "La ricerca dei contenuti in rete". En M. Morcellini. La cultura della tesi. Lecce: La Biblioteca Pensa Multimedia, pp. 121-132.

MELTZER, B., PETRAS, J. y REYNOLDS, L. (1980). Interazionismo simbolico. Milano: Franco Angeli.

MENGALDO, P. V. (1978). Poeti italiani del Novecento. Milano: Mondadori.

MISAN-MONTEFIORE, J. (2007). "La maschera e il volto nelle opere di Proust e Pirandello", en Studi francesi, 152: 390-403.

MONTI, E. (2008). Ritratti di interazionisti simbolici americani. Milano: Franco Angeli.

NOYA, J. (2004). "Teoría e investigación en la sociología española contemporánea", en Política y Sociedad, vol. 41, 2: 163-174.

OCAMPO BARRIO, P. y PÉREZ MEJÍA, A. N. (2010). "Creencias y percepciones de las personas obesas acerca de la obesidad", en Semergen, 6: 325-331.

OLIVENA RIVERA, E. (2006). "La escuela pública como representación simbólica popular", en Revista Iberoamericana de Educación, vol. 40, 4: 1-14.

PERELLÓ OLIVER, S. (2009). Metodología de la investigación social. Madrid: Dykinson.

PERLO, C. (2006). "Aportes del Interaccionismo simbólico a las teorías de la organización", en Invenia, pp. 89-107.

PERROTTA, R. (2005). Cornici, specchi, maschere. Interazionismo simbolico e comunicazione. Bologna: Clueb.

- (2010). Un cuore di farfalla. Milano: Franco Angeli.

PICÓ LÓPEZ, J. (1997). “Teoría social: la relación entre Europa y Estados Unidos”, en Reis, 80: 95109.

PICÓ LÓPEZ, J. y SERRA, I. (2010). La Escuela de Chicago de Sociología. Madrid: Siglo XXI.

PIOVANI, J. (2011). "La escuela de Chicago y los enfoques cualitativos: términos y conceptos metodológicos", en Papers, 96: 245-258.

PONS DÍEZ, X. (2010). "La aportación a la psicología social del interaccionismo simbólico", en EduPsykhé, vol. 9, 1: 23-41.

RAUTY, R. (2008). "Introduzione". En H. Blumer Interazionismo simbolico. Bologna: il Mulino.

RIZO GARCÍA, M. (2011). "De personas, rituales y máscaras. Ervin Goffman y sus aportes a la comunicación interpersonal", en Quórum Académico, nº 8, 15: 78-94.

ROCK, P. (1979). The Making of Symbolic Interactionism. Londres: The Macmillan Press.

ROMANIA, V. (2004). Farsi passare per italiani. Roma: Carocci.

- (2012). Ruoli, identitá, interazioni. Napoli: Liguori.

SAGOT, M. (1994). "Marxismo, interaccionismo simbólico y la opresión de la mujer", en Ciencias Sociales, 63: 129-140.

SBARBERI, F. (1999). L'utopia della libertá uguale. Torino: Bollati Boringhieri.

STRANIERO, G. (2004). Faccia a faccia. Interazione sociale e osservazione partecipante nell'opera di Erving Goffman. Torino: Bollati Boringhieri.

TOMASINI, M. E. (2010). "Un viejo pensador para resignificar una categoría psicosocial: George Mead y la socialización", en Athenea Digital, 17: 137-156.

UÑA JÚAREZ, O. (2009). Nuevos ensayos de sociología y comunicación. Madrid: Universitas. 
WALLACE, R. y WOLF, A. (1985). La Teoria Sociologica contemporanea. Bologna: Il Mulino. ZABLUDOVSKY KUPER, G. (2009). Intelectuales y burocracia. Vigencia de Max Weber. Barcelona: Anthropos.

ZUNZ, O. (2002). Perché il secolo americano. Bologna: Il Mulino.

\section{Breve currículo:}

\section{Giuliano Tardivo}

Profesor visitante de la URJC. Licenciado cum laude en Ciencias Políticas por la Universidad de Padua (título homologado por la UCM). Master en Instituciones locales e integración europea y en Gestión e Investigación de la Comunicación empresarial.

\section{Maximiliano Fernández Fernández}

Profesor contratado doctor de la Universidad Rey Juan Carlos de Madrid. Licenciado y doctor en Ciencias de la Información por la Universidad Complutense de Madrid y en Ciencias Políticas y Sociología por la Universidad Pontificia de Salamanca (Instituto León XIII de Madrid). Autor de una veintena de libros sobre comunicación y sociología. 